\title{
Extracapsular extension on multiparametric magnetic resonance imaging better predicts pT3 disease at radical prostatectomy compared to perineural invasion on biopsy
}

Luke Griffiths ${ }^{1}$; Srinath Kotamarti ${ }^{1,2}$; David Mikhail ${ }^{1,3}$; Joseph Sarcona ${ }^{3}$; Ardeshir R. Rastinehad $^{1}$; Robert Villani ${ }^{1}$; Jessica Kreshover ${ }^{1}$; Simon J. Hall ${ }^{1}$; Manish A. Vira ${ }^{1}$; Michael J. Schwartz ${ }^{1}$; Lee Richstone ${ }^{1,3}$

${ }^{1}$ Smith Institute for Urology, Northwell Health, New Hyde Park, NY, United States; ${ }^{2}$ Department of Urology, Maimonides Medical Center, Brooklyn, NY, United States; ${ }^{3}$ Department of Urology, Lenox Hill Hospital, New York, NY, United States

Cite as: Griffiths L, Kotamarti S, Mikhail D, et al. Extracapsular extension on multiparametric magnetic resonance imaging better predicts $\mathrm{pT} 3$ disease at radical prostatectomy compared to perineural invasion on biopsy. Can Urol Assoc J 2021 January 4; Epub ahead of print. http://dx.doi.org/10.5489/cuaj.6909

Published online January 4, 2021

$* * *$

\section{Abstract}

Introduction: Risk assessment for non-organ-confined prostate cancer $(\mathrm{PCa})$ is important in the surgical planning for radical prostatectomy (RP). Perineural invasion (PNI) on prostate biopsy has been associated with adverse pathological outcomes at prostatectomy. Similarly, the identification of suspected extracapsular extension (ECE) on multiparametric magnetic resonance imaging (mpMRI) has been shown to predict non-organ-confined disease. However, no prior study has compared these factors in predicting adverse pathology at prostatectomy. We evaluated mpMRI ECE and prostate biopsy PNI on multivariable analysis to determine their ability to predict pathological stage at time of RP.

Methods: We retrospectively investigated the prostatectomy database at our institution to identify men who underwent prostate biopsy with pre-biopsy mpMRI and subsequent RP from 2013-2017. Multivariable regression analysis was performed to compare the association of mpMRI ECE (mECE) and PNI on prostate biopsy on the likelihood of finding pT3 disease on pathology post-prostatectomy.

Results: Of a total 454 RP between 2013 and 2017, 191 patients met our inclusion criteria. Stage pT2 and pT3+ were found in $120(62.8 \%)$ and $71(37.2 \%)$ patients, respectively. Patients with mECE had 4.84 cumulative odds of worse pathological stage on RP $(p=0.045)$ compared to PNI on biopsy, which showed cumulative odds of $2.25(\mathrm{p}=0.048)$. When controlling only for those 
patients without PNI, mECE was still found to be a significant predictor of pT3 disease at RP $(\mathrm{p}=0.030)$. However, in patients without $\mathrm{mECE}$, PNI was not significant $(\mathrm{p}=0.062)$.

Conclusions: While $\mathrm{mECE}$ and biopsy PNI were both associated with worse pathological stage on RP, mECE had significantly higher cumulative odds compared to PNI. The significant predictive ability of $\mathrm{mECE}$ adds further clinical value to the use of mpMRI in PCa management. While validation in a larger cohort is required, these factors have important clinical implications with regards to early diagnosis of advanced disease and surgical planning.

\section{Introduction}

In patients with localized prostate cancer, nerve-sparing surgery offers improved post-operative functional outcomes and is considered the standard approach to radical prostatectomy (RP). ${ }^{1,2}$ Implementing a nerve-sparing approach, however, is not appropriate for every case and the decision to nerve spare is influenced by the estimated probability of extraprostatic extension (EPE) on prostatectomy. ${ }^{3,4}$ To optimize surgical and treatment planning, risk assessment for features of non-organ-confined disease is performed through nomogram assessment of preoperative clinical features, such as preoperative PSA, biopsy Gleason score, and clinical stage. $^{5-9}$ However, preoperative estimates are often inaccurate, and in patients where the tumor extends outside of the prostate, the risk of positive surgical margin and adverse pathology may be particularly high. ${ }^{10}$

In an effort to improve existing predictive risk models, additional preoperative parameters have been studied for their utility. Perineural invasion is defined as the tracking of tumor cells along or around nerve fibers; in prostate cancer this extension is commonly reported on prostate biopsy pathology. ${ }^{11,12}$ While not traditionally utilized in nomogram assessment, perineural invasion (PNI) on prostate biopsy (PBx) is associated with EPE at RP as well as margin positivity and biochemical recurrence. ${ }^{13-16}$

Multi-parametric magnetic resonance imaging (mpMRI) is a PCa diagnostic tool that is increasingly popular and is being utilized to direct prostate biopsy and surgical planning. ${ }^{17}$ Preoperative mpMRI is useful for assessing the presence of significant cancer, predicting organ confined disease, and assessing seminal vesicle invasion. ${ }^{17}$ While having a low to moderate sensitivity, mpMRI has achieved high specificity and positive predictive values of up to $90 \%$ in detecting extracapsular extension and seminal vesicle invasion. ${ }^{18-22}$ Interestingly, while both PNI on PBx and mpMRI extracapsular extension (mECE) have been shown as independent predictors of adverse outcomes and non-organ-confined disease, no prior study has compared these factors together. To query whether PNI is a significant prognostic variable in the era of mpMRI, we evaluated and compared the ability of mECE and PNI on prostate biopsy to predict advanced pathological stage at time of RP. 


\section{Methods}

\section{Patient population}

We retrospectively investigated the prostatectomy database at our institution to identify men who underwent radical prostatectomy from July 2013 through March 2017. Patients were included if they received an mpMRI and subsequent prostate biopsy before radical prostatectomy at our institution. Patients were excluded if they had missing information, incomplete mpMRI, if biopsy or mpMRI were conducted at an outside institution, or history of alternative or previous treatment for prostate cancer such as radiation therapy, cryoablation, or any neoadjuvant therapy. A subgroup analysis was performed to ascertain if mECE or PNI would reach significance in prediction of pT3 when excluding all mECE patients and then all PNI patients from the original cohort. All staging was assigned according to the 2017 American Joint Committee on Cancer (AJCC) staging criteria. ${ }^{23}$

\section{Protocol for mpMRI}

Patients were referred for mpMRI in the setting of elevated PSA or PSA density. Urologists at our institution routinely obtained mpMRI prior to biopsy to utilize a fusion approach and for potential surgical planning. All patients underwent mpMRI (3T Verio, Siemens, Germany ®) of

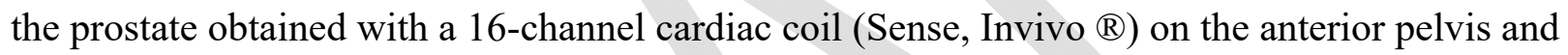

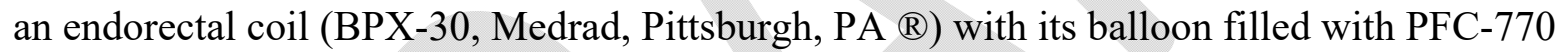
(3M, St. Paul, MN ®). Sequences obtained included tri-planar T2-weighted, diffusion-weighted imaging (DWI) (b-values 0, 500, 1000, and 1500) and separate b-2000 and dynamic contrast enhanced (DCE) sequences. All mpMRI were read by experienced genitourinary radiologists, who assigned risk scores using the PI-RADS v1 and PI-RADS v2 scoring system. If multiple suspicious lesions were present, the highest PI-RADS score reported was used. Extra-capsular extension on MRI was stratified based on radiology report into mECE present, mECE suspicious, or mECE absent. mECE 'present' was assigned if ECE was clearly seen, as described by the radiologist, with gross extension of disease. mECE 'suspicious' was assigned to reports including descriptions of "abutment", capsular "bulging", or "microscopic invasion cannot be ruled out”. mECE 'absent' was assigned when there was clearly no evidence of ECE on MRI.

\section{Protocol for prostate biopsy}

All biopsies were performed via transrectal technique by experienced urologists at our institution. MRI-Targeted images were processed on a Dynacad workstation (Invivo, Gainesville, FL $\left.{ }^{\circledR}\right)$. MR/TRUS image fusion was performed using UroNav ${ }^{\circledR}$ software in conjunction with an IU-22 (Philips Health Care, Best Netherlands) end-fire ultrasound probe. During biopsies of the lesions, one core was obtained in the axial and sagittal planes for a total of two cores per lesion followed by 12-core systematic biopsy as per our institution's protocol for MRI fusion biopsy. Patients without a targetable lesion identified on MRI received a standard 
12-core transrectal ultrasound (TRUS) biopsy whereby 2 cores, medial and lateral, are taken from each sextant region in a standard fashion. All biopsy pathology slides were reviewed by our institution's experienced genitourinary pathologist.

\section{Statistical methods}

Univariable analysis was performed to determine if there were differences in age, PSA at MRI, prostate volume, PIRADS score, and ECE between pT2 and pT3+. A Mann-Whitney U test was run for non-normally distributed continuous variables and chi square was run for nominal parameters. A cumulative odds ordinal logistic regression with proportional odds was run to determine the effect of 6 variables on pathological cancer staging after radical prostatectomy. These variables included age, PNI on PBx, mECE, PI-RADS, PSA, and MRI prostate volume. A binomial logistic regression analysis was performed on all the variables to ascertain the likelihood of subjects having positive margins. PI-RADS, PSA, age, and prostate volume were analyzed as continuous variables. Proportional odds were assessed by a full likelihood ratio test comparing the fitted model to a model with varying location parameters. The model fit was tested through a deviance goodness-of-fit test. Collinearity of the independent variables was assessed and ruled out through inspection of correlation coefficients and tolerance/VIF values. SPSS 24.0.0 for Windows (SPSS Inc., Chicago, IL, USA) was used for all statistical analysis with $p<0.05$ considered statistically significant.

\section{Results}

During the study period, 454 patients underwent RP at our intuition. 191 patients met our inclusion criteria with baseline characteristics shown on Table 1. Stage pT2 and pT3 were found in $120(62.8 \%)$ and $71(37.2 \%)$ patients after prostatectomy. Biopsy pathology classified 28 (14.7\%), 81 (42.4\%), and $82(42.9 \%)$ patients into Gleason grade group 1, 2, and 3-5 respectively. Prostate needle biopsy PNI was positive in $55(22.8 \%)$ and absent in $136(71.2 \%)$ of patients. On mpMRI, mECE was found in 12 (6.3\%), suspicious mECE in 32 (16.8\%), and mECE absent in 147 (77\%) patients (Table 1).

Multiparametric MRI findings for mECE and suspicious mECE were compared to final pathology to determine sensitivity, specificity, PPV, and NPV of detecting pT3 disease (Table 2). mECE present had sensitivity, specificity, PPV, and NPV of 14.1\% (95\% CI 7.3-24.8), 98.3\% (95\% CI 93.5-99.7), 83.3\% (95\% CI 50.9-97.1), and 65.9\% (95\% CI 58.4-72.7), respectively. On the other hand, mECE suspicious resulted in a sensitivity, specificity, PPV and NPV of $23.9 \%$ (95\% CI 14.9-35.8), 87.5\% (95\% CI 79.9-92.6), 53.1\% (95\% CI 35.0-70.5), and 66\% (95\% CI 58.1-73.2), respectively.

Univariable analysis revealed statistical significance between pT2 and pT3+ groups with regards to PSA, PNI, PIRADS, and ECE. Age and prostate volumes did not reach significance (Table 1). On multivariable analysis, PNI on PBx predicted 2.25 times higher odds of pT3 
disease [ $\mathrm{p}=0.045,95 \%$ CI 1.101 - 3.431]. Similarly, presence of extracapsular extension on MRI also predicted pT3 disease with an odds of 4.84 times [p $=0.048,95 \%$ CI $1.02-23.17]$. However, ECE suspicious on MRI did not significantly predict advanced stage at prostatectomy compared to absent ECE on MRI $(p=0.814)$. Increased PI-RADS and PSA at MRI were both associated with an increase in the odds of predicting advanced stage PCa on RP (OR of 2.20, 95\% CI [1.29 - 3.752] and 1.10, 95\% CI [1.012 - 1.184], respectively) as seen in Table 3. Age was not associated with an increased odds of predicting pathological stage $(p=0.542)$. When assessing for surgical margin status on binary logistic regression, only mECE present was statistically associated with an increased likelihood of exhibiting positive surgical margins with an OR of 6.57 [ $p=0.031,95 \%$ CI $(1.19-36.15)$; Table 4]. Fifty (90.9\%) patients with PNI did not have mECE; of these, 28 (56.0\%) were pT3.

In subgroup analysis, when controlling only for those patients without PNI $(n=136), 7$ (5.1\%) patients had mECE; of these, $5(71.4 \%)$ were pT3. Within this cohort, mECE was still found to be a significant predictor of pT3 disease at RP ( $\mathrm{p}=0.03)$. When analyzing mECE absent patients $(\mathrm{n}=174), 50(90.9 \%)$ had PNI on $\mathrm{pBx}$; of these, $28(56.0 \%)$ were pT3 PNI on PBx $(n=50)$. This cohort, however, did not significantly predict $\mathrm{pT} 3$ disease $(\mathrm{p}=0.062)$.

\section{Discussion}

To our knowledge, this is the first study to compare PNI and mECE together in a predictive model for at least pT3 disease at time of RP. In particular, we found mECE to have an increased likelihood of predicting pT3 compared to that of PNI on prostate biopsy. Additionally, in our population mECE also was shown to be an independent predictor of positive surgical margins. Importantly, when PNI was present but there was no ECE on MRI, there was no significant increase in pT3 disease.

Historically, preoperative risk stratification methods have implemented certain clinical and laboratory elements to predict oncologic outcomes. Common methods such as the Partin Tables or D'Amico Risk Stratification for prostatectomy oncologic outcomes include the use of PSA, Gleason score, and clinical staging. ${ }^{5,9,24}$ While these stratification methods do not use PNI on PBx, PNI has been shown to be associated with adverse pathological outcomes at time of RP. ${ }^{13,14,16}$ A recent retrospective review by Celik et al. stratified post-prostatectomy patients based on RP pathological stage, PNI presence on PBx, and D'Amico risk stratification. The authors suggested the presence of PNI is an important predictive marker due to positive correlation with local invasive disease after RP in all stratification groups, especially highlighting that those with intermediate D'Amico risk and positive PNI should have increased suspicion for locally advanced disease. ${ }^{13}$ An earlier review conducted by Delancey et al. in 2013, also sought to determine the effect of PNI on adverse pathological features at time of RP, as well as to determine survival outcomes in these patients. On multivariable models controlling for biopsy Gleason score, PSA, and clinical stage, they found patients with PNI on PBx had 2.27, 
2.64, and 1.64 increased odds of having extraprostatic extension, seminal vesical involvement, and positive surgical margins, respectively. Additionally, their analysis of survival outcomes showed decreased disease free and overall survival times. ${ }^{14}$ While our results for predicting pT3 are in line with these findings showing similar increased odds of 2.25 , association of positive surgical margins did not achieve significance. This is likely related to controlling for different factors between our models (Table 3).

In another study examining PNI, Cozzi et al. conducted a systematic review and metaanalysis in which they also found patients with PNI on PBx had a significant association with pT3 tumors at time of RP. ${ }^{16}$ Of their 7981 patients reported, they found PNI positivity on PBx to be $15.8 \%$, and in patients with PNI, pT3 pathology was found in $53.4 \%$ of RP specimens. These findings agree wither similar studies which showed just over half of patients with PNI having pT3. ${ }^{14,15}$ While the present study found an increased PNI positivity rate of $22.8 \%$, this is similar to previously published rates of PNI. ${ }^{13-15,25}$ Our findings of an increased rate at $60 \%$ of PNI patients having pT3 disease may be attributed to our population having an overall higher rate of pT3 at $37.1 \%$ compared to $27.9 \%$ as seen in Cozzi et al. Clearly, presence of PNI on PBx has been implicated as a predictor of non-organ confined disease, and consequently, some have agreed nerve sparing techniques should not be performed in such patients.

In our cohort, we looked to evaluate if the presence of PNI on PBx holds significance in the age of high-resolution imaging. Specifically, would mpMRI prediction of ECE exceed PNI on PBx as a predictor of non-organ confined disease? Furthermore, does the presence of PNI even maintain predictive significance when mpMRI fails to show mECE?

Our study demonstrated mECE was, in fact, a better predictor of adverse pathology compared to PNI on PBx. The continued rise in mPMRI usage for pre-surgical evaluation and management of prostate cancer has allowed for further exploration into how these tools can be incorporated in predicting patient outcomes. While the impact of PIRADS has been well studied, mECE provides a logical tool to identify patients at risk for adverse postoperative pathology. In a study examining whether findings of $\mathrm{MECE}$ were associated with upstaging or upgrading at prostatectomy by Hedge et al., they followed the MECE T3 staging based on findings of asymmetric bulging, irregular margins, or direct extension of the lesion into periprostatic fat or neurovascular bundle staging as reported previously by Roethke et al. ${ }^{26,27}$ On multivariable logistic regression, they found a 4.81 adjusted odds ratio for increased likelihood of pT3 after RP compared to pT2. These findings are in line with our study results of 4.84 increased odds of pT3 compared to pT2 when $\mathrm{mECE}$ is present. Interestingly, our $\mathrm{mECE}$ suspicious group did not achieve significance for independently predicting pT3 at time of RP. This finding perhaps could be attributed to the inclusion of PIRADS scoring into the regression model.

Utilizing mECE in predictive models has also shown a potential benefit. Dominguez et al. implemented mECE findings using a $1.5 \mathrm{~T} \mathrm{mpMRI}$ in patients with intermediate and high D'Amico risk to establish an AUC of 0.7 for predicting non-organ confined disease at time of 
RP. ${ }^{18}$ Taking a step further, when comparing these mECE against the Partin tables, Gupta et al. found that mECE had an AUC of 0.82 compared to those of the Partin table's at $0.62(p=0.04)$ in terms of predicting adverse pathologic stage. ${ }^{28}$ Interestingly, sensitivity of mECE in predicting pT3 using a 3.0T MRI scanner is variable and generally low across literature ranging from $35-74 \%$, and our study was no different, reporting sensitivities of $14.1 \%$ and $23.9 \%$ for mECE present and mECE suspicious, respectively. However, specificity is consistently reported as robust for the prediction of pT3 with $\mathrm{mECE}$, as we confirmed in our study, finding a specificity of $98.3 \%$ for mECE presence..$^{22,29,30}$

The present study was not without its limitations. As a retrospective study, it is subject to inherent biases including selection bias as a possible explanation for our higher observed pT3 rate at RP. Evaluating for both PNI and mECE decreased the included sample size, resulting in large confidence intervals, specifically for mECE. Further validation with a larger cohort is warranted. Unlike the PIRADS scoring system, reporting for mECE does not follow a strict standardized procedure and is vulnerable to subjectivity and interobserver variability between radiologists. Evaluating $\mathrm{mECE}$ presents a challenge due to inherent subjectivity of proper characterization; although similar, a non-standardized and heterogenous approach to MRI staging amongst studies exists in the literature. ${ }^{18,22,26,28}$ Unfortunately, there does not exist a standardized stratification for $\mathrm{mECE}$ at this time. Furthermore, there are a plethora of possible descriptions for addressing ECE that fall in between "no ECE" and "ECE present." We attempted to account for this by stratifying the groups to separately analyze the less straight forward 'mECE suspicious' reported lesions. Although during this time period, many institutions did not routinely obtain mpMRI prior to prostate biopsy, our institution routinely obtained mpMRI prior to biopsy on patients with elevated PSA to allow for fusion biopsy approach. When challenged, we were often able to obtain high insurance approval rates through peer-to-peer authorization discussions.

Our findings support $\mathrm{mECE}$ as having the potential to significantly predict the presence of pT3 disease on radical prostatectomy. When excluding all patients with mECE present, PNI positivity on its own did not significantly predict pT3 disease. Conversely, when all PNI patients were excluded, mECE still retained its significance in predicting pT3. Although the development of an accepted standardized reporting system for extracapsular extension is necessary for inclusion into a nomogram for risk assessment, our study further supports the use of MRI preoperatively in perioperative surgical and treatment planning for organ confined prostate cancer.

\section{Conclusions}

While mECE and PNI on PBx were both associated with worse pathological stage on RP, mECE had significantly higher cumulative odds compared to PNI. The significant predictive ability of ECE on multivariable analysis, and when controlling for only non-PNI patients, adds further clinical value to the use of mpMRI in prostate cancer management. In patients with no suspicion 
for ECE on mpMRI, PNI is not associated with increased risk for T3 disease. This has potential implications when selecting patients for nerve sparing prostatectomy. While validation in a larger cohort is required, these factors may have important clinical implications with regards to early diagnosis of advanced disease, while also highlighting the necessity for selective nerve sparing to be further explored. 


\section{References}

1. Heidenreich A, Bastian PJ, Bellmunt $\mathrm{J}$, et al. EAU guidelines on prostate cancer. part 1: screening, diagnosis, and local treatment with curative intent-update 2013. Eur Urol. 2014;65(1):124-137.

2. Nguyen LN, Head L, Witiuk K, et al. The Risks and Benefits of Cavernous Neurovascular Bundle Sparing during Radical Prostatectomy: A Systematic Review and Meta-Analysis. J Urol. 2017;198(4):760-769.

3. Tanguturi SK, Chen MH, Loffredo M, Richie JP, D'Amico AV. Evaluating the Impact of PSA as a Selection Criteria for Nerve Sparing Radical Prostatectomy in a Screened Cohort. Prostate Cancer. 2014;2014:395078.

4. Wright JL, Izard JP, Lin DW. Surgical management of prostate cancer. Hematol Oncol Clin North Am. 2013;27(6):1111-1135, vii.

5. Partin AW, Yoo J, Carter HB, et al. The use of prostate specific antigen, clinical stage and Gleason score to predict pathological stage in men with localized prostate cancer. $J$ Urol. 1993;150(1):110-114.

6. Kattan MW, Eastham JA, Stapleton AM, Wheeler TM, Scardino PT. A preoperative nomogram for disease recurrence following radical prostatectomy for prostate cancer. $J$ Natl Cancer Inst. 1998;90(10):766-771.

7. Kattan MW, Wheeler TM, Scardino PT. Postoperative nomogram for disease recurrence after radical prostatectomy for prostate cancer. J Clin Oncol. 1999;17(5):1499-1507.

8. D'Amico AV, Whittington R, Malkowicz SB, et al. Combination of the preoperative PSA level, biopsy gleason score, percentage of positive biopsies, and MRI T-stage to predict early PSA failure in men with clinically localized prostate cancer. Urology. 2000;55(4):572-577.

9. Tosoian JJ, Chappidi M, Feng Z, et al. Prediction of pathological stage based on clinical stage, serum prostate-specific antigen, and biopsy Gleason score: Partin Tables in the contemporary era. BJU Int. 2017;119(5):676-683.

10. Retèl VP, Bouchardy C, Usel M, et al. Determinants and effects of positive surgical margins after prostatectomy on prostate cancer mortality: a population-based study. BMC Urol. 2014;14:86.

11. Hassan MO, Maksem J. The prostatic perineural space and its relation to tumor spread: an ultrastructural study. Am J Surg Pathol. 1980;4(2):143-148.

12. Liebig C, Ayala G, Wilks JA, Berger DH, Albo D. Perineural invasion in cancer: a review of the literature. Cancer. 2009;115(15):3379-3391.

13. Celik S, Bozkurt O, Demir O, et al. Effects of perineural invasion in prostate needle biopsy on tumor grade and biochemical recurrence rates after radical prostatectomy. Kaohsiung J Med Sci. 2018;34(7):385-390.

14. DeLancey JO, Wood DP, He C, et al. Evidence of perineural invasion on prostate biopsy specimen and survival after radical prostatectomy. Urology. 2013;81(2):354-357.

15. Gorin MA, Chalfin HJ, Epstein JI, Feng Z, Partin AW, Trock BJ. Predicting the risk of non-organ-confined prostate cancer when perineural invasion is found on biopsy. Urology. 2014;83(5):1117-1121. 
16. Cozzi G, Rocco BM, Grasso A, et al. Perineural invasion as a predictor of extraprostatic extension of prostate cancer: a systematic review and meta-analysis. Scand J Urol. 2013;47(6):443-448.

17. Fulgham PF, Rukstalis DB, Turkbey IB, et al. AUA Policy Statement on the Use of Multiparametric Magnetic Resonance Imaging in the Diagnosis, Staging and Management of Prostate Cancer. J Urol. 2017;198(4):832-838.

18. Dominguez C, Plata M, Cataño JG, et al. Diagnostic accuracy of multiparametric magnetic resonance imaging in detecting extracapsular extension in intermediate and high - risk prostate cancer. Int Braz J Urol. 2018;44(4):688-696.

19. Jeong IG, Lim JH, You D, et al. Incremental value of magnetic resonance imaging for clinically high risk prostate cancer in 922 radical prostatectomies. $J$ Urol. 2013;190(6):2054-2060.

20. Lista F, Gimbernat H, Cáceres F, Rodríguez-Barbero JM, Castillo E, Angulo JC. Multiparametric magnetic resonance imaging for the assessment of extracapsular invasion and other staging parameters in patients with prostate cancer candidates for radical prostatectomy. Actas Urol Esp. 2014;38(5):290-297.

21. Pinaquy JB, De Clermont-Galleran H, Pasticier G, et al. Comparative effectiveness of [(18) F]-fluorocholine PET-CT and pelvic MRI with diffusion-weighted imaging for staging in patients with high-risk prostate cancer. Prostate. 2015;75(3):323-331.

22. Cerantola Y, Valerio M, Kawkabani Marchini A, Meuwly JY, Jichlinski P. Can 3T multiparametric magnetic resonance imaging accurately detect prostate cancer extracapsular extension? Can Urol Assoc J. 2013;7(11-12):E699-703.

23. Amin MB, Greene FL, Edge SB, et al. The Eighth Edition AJCC Cancer Staging Manual: Continuing to build a bridge from a population-based to a more "personalized" approach to cancer staging. CA Cancer J Clin. 2017;67(2):93-99.

24. D'Amico AV, Whittington R, Malkowicz SB, et al. Biochemical outcome after radical prostatectomy, external beam radiation therapy, or interstitial radiation therapy for clinically localized prostate cancer. JAMA. 1998;280(11):969-974.

25. Bismar TA, Lewis JS, Vollmer RT, Humphrey PA. Multiple measures of carcinoma extent versus perineural invasion in prostate needle biopsy tissue in prediction of pathologic stage in a screening population. Am J Surg Pathol. 2003;27(4):432-440.

26. Hegde JV, Chen MH, Mulkern RV, Fennessy FM, D'Amico AV, Tempany CM. Preoperative 3-Tesla multiparametric endorectal magnetic resonance imaging findings and the odds of upgrading and upstaging at radical prostatectomy in men with clinically localized prostate cancer. Int J Radiat Oncol Biol Phys. 2013;85(2):e101-107.

27. Roethke MC, Lichy MP, Kniess M, et al. Accuracy of preoperative endorectal MRI in predicting extracapsular extension and influence on neurovascular bundle sparing in radical prostatectomy. World J Urol. 2013;31(5):1111-1116.

28. Gupta RT, Faridi KF, Singh AA, et al. Comparing 3-T multiparametric MRI and the Partin tables to predict organ-confined prostate cancer after radical prostatectomy. Urol Oncol. 2014;32(8):1292-1299. 
29. Somford DM, Hamoen EH, Fütterer JJ, et al. The predictive value of endorectal 3 Tesla multiparametric magnetic resonance imaging for extraprostatic extension in patients with low, intermediate and high risk prostate cancer. J Urol. 2013;190(5):1728-1734.

30. Boesen L, Chabanova E, Løgager V, Balslev I, Mikines K, Thomsen HS. Prostate cancer staging with extracapsular extension risk scoring using multiparametric MRI: a correlation with histopathology. Eur Radiol. 2015;25(6):1776-1785.

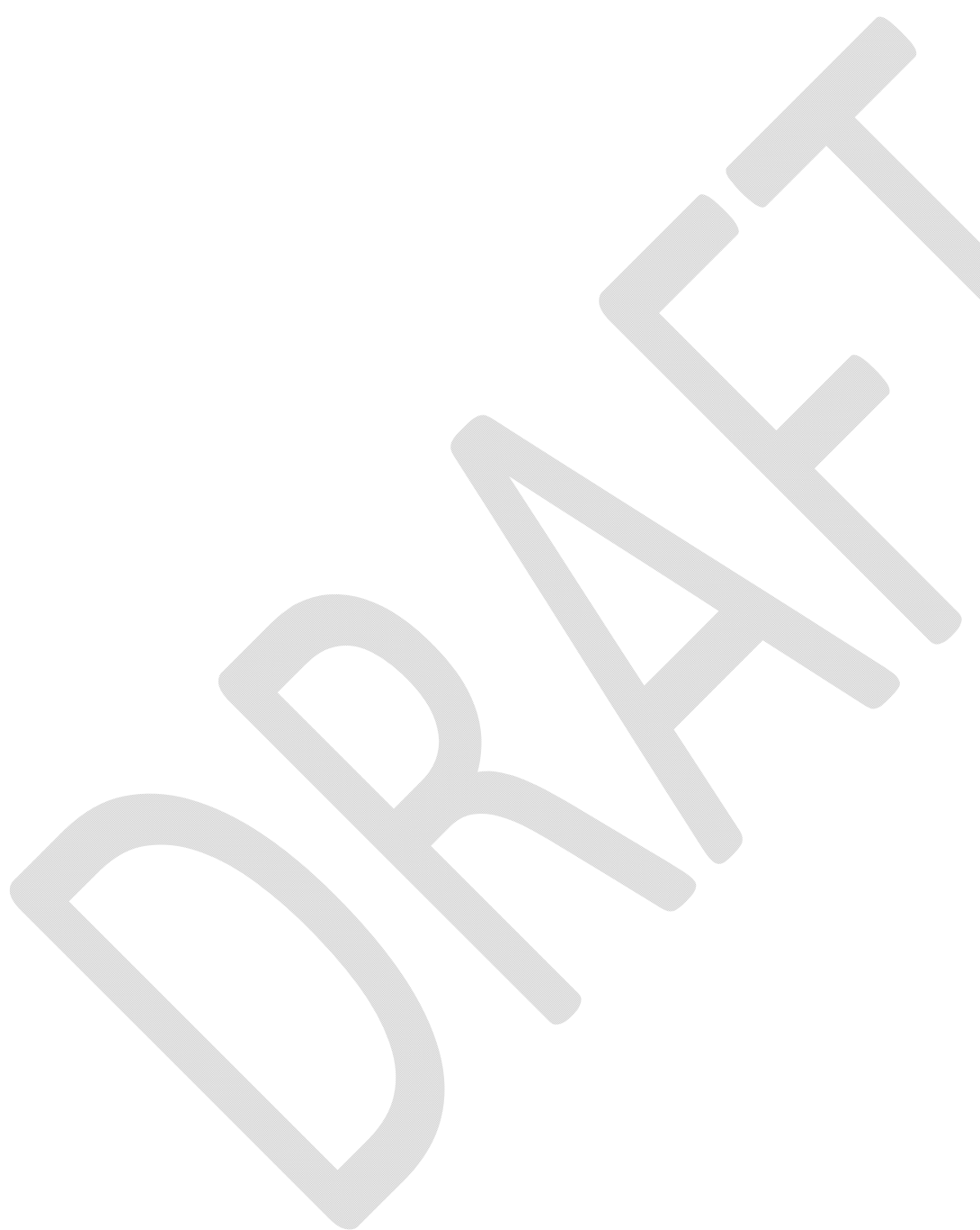




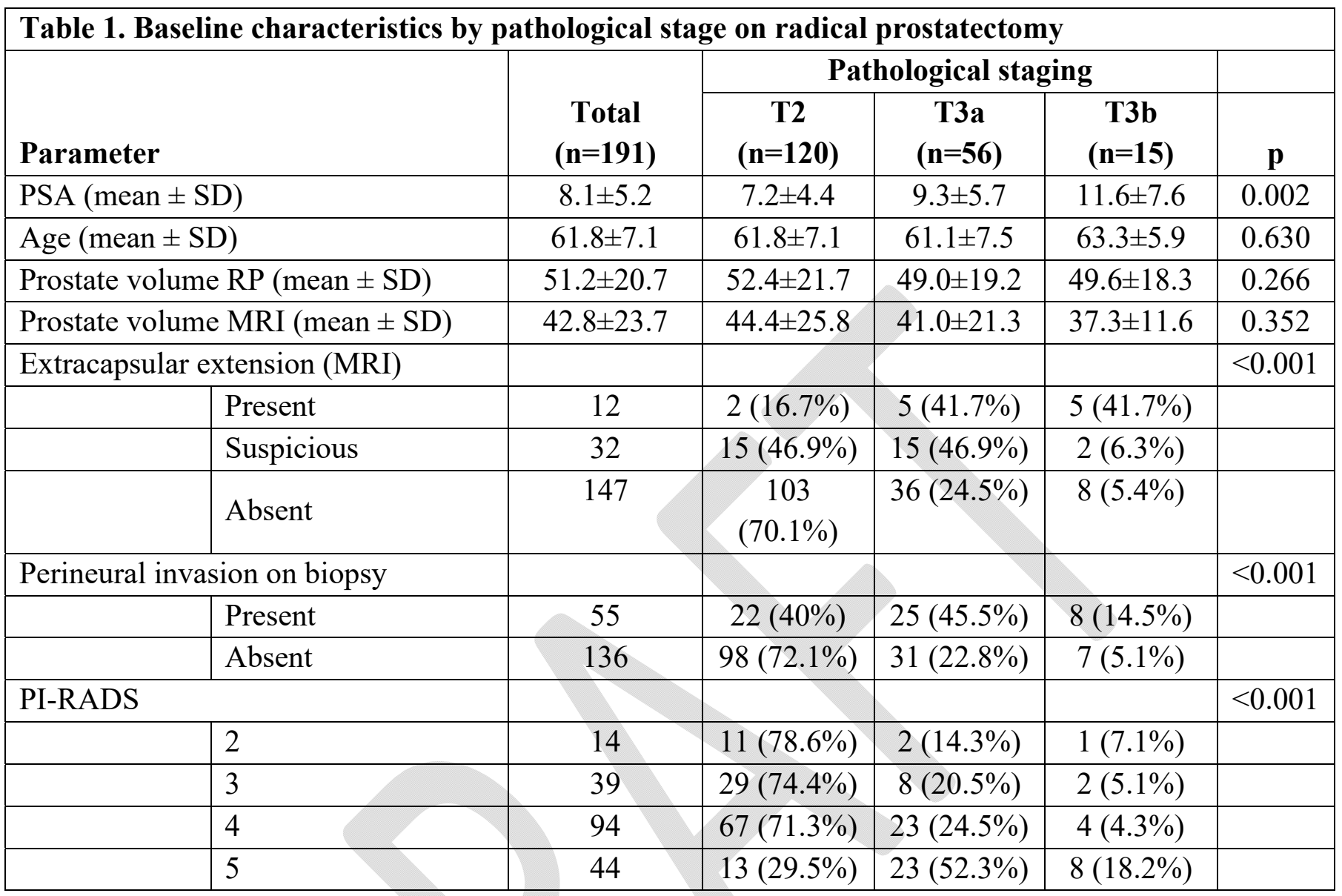

p-values reflect univariable analysis comparing parameters to pT2 and pT3 disease. ECE: extracapsular extension; MRI: magnetic resonance imaging; PI-RADS: Prostate Imaging-Reporting and Data System; PSA: prostate-specific antigen; RP: radical prostatectomy; SD: standard deviation. 
ECE vs. PNI in predicting pT3 disease in radical prostatectomy

\begin{tabular}{|c|c|}
\hline Parameter & Percentage (95\% CI) \\
\hline \multicolumn{2}{|l|}{ mECE present } \\
\hline Sensitivity & $14.1 \%(7.3-24.8)$ \\
\hline Specificity & $98.3 \%(93.5-99.7)$ \\
\hline PPV & $83.3 \%(50.9-97.1)$ \\
\hline NPV & $65.9 \%(58.4-72.7)$ \\
\hline \multicolumn{2}{|c|}{ mECE suspicious } \\
\hline Sensitivity & $23.9 \%(14.9-35.8)$ \\
\hline Specificity & $87.5 \%(79.9-92.6)$ \\
\hline PPV & $53.1 \%(35.0-70.5)$ \\
\hline NPV & $66 \%(58.1-73.2)$ \\
\hline \multicolumn{2}{|l|}{ PBx PNI } \\
\hline Sensitivity & $46.5 \%(34.6-58.7)$ \\
\hline Specificity & $81.7 \%(73.6-88.1)$ \\
\hline PPV & $60.0 \%(48.8-70.2)$ \\
\hline NPV & $72.1 \%(67.1-76.5)$ \\
\hline
\end{tabular}

CI: confidence interval; mECE: multiparametric MRI extracapsular extension; NPV: negative predictive value; PBx PNI: prostate biopsy perineural invasion; PPV: positive predictive value. 


\begin{tabular}{|c|c|c|}
\hline Parameter & OR (95\% CI) & $\mathbf{p}$ \\
\hline PNI present (Bx) & $2.25(1.02-4.97)$ & 0.045 \\
\hline \multicolumn{3}{|l|}{ PNI absent $(\mathrm{Bx})$} \\
\hline ECE present (MRI) & $4.84(1.01-23.17)$ & 0.048 \\
\hline ECE suspicious (MRI) & $0.88(0.29-2.66)$ & 0.814 \\
\hline ECE absent (MRI) & $*$ & \\
\hline Age & $1.02(0.94-1.07)$ & 0.542 \\
\hline Prostate volume (MRI) & $0.99(0.97-1.00)$ & 0.135 \\
\hline PSA (MRI) & $\mathbf{1 . 1 0}(1.01-1.18)$ & 0.023 \\
\hline PI-RADS & $2.20(1.29-3.75)$ & 0.004 \\
\hline
\end{tabular}

*Variable used as referent. CI: confidence interval; ECE: extracapsular extension; MRI: magnetic resonance imaging; OR: odds ratio; PI-RADS: Prostate Imaging-Reporting and Data System; PNI: perineural invasion; PSA: prostate-specific antigen.

\begin{tabular}{|c|c|c|}
\hline Parameter & OR $(95 \%$ CI $)$ & $\mathbf{p}$ \\
\hline Age & $1.02(0.97-1.08)$ & 0.436 \\
\hline PNI (Bx) & $1.70(0.75-3.86)$ & 0.209 \\
\hline PSA (MRI) & $1.04(0.96-1.13)$ & 0.328 \\
\hline Prostate volume (MRI) & $0.99(0.98-1.01)$ & 0.610 \\
\hline ECE not present (MRI) & $*$ & \\
\hline ECE suspicious (MRI) & $2.18(0.78-6.06)$ & 0.135 \\
\hline ECE present & $6.57(1.19-36.15)$ & 0.031 \\
\hline
\end{tabular}

*Variable used as referent. CI: confidence interval; ECE: extracapsular extension; MRI: magnetic resonance imaging; OR: odds ratio; PNI: perineural invasion; PSA: prostate-specific antigen. 